Discrete Comput Geom 31:1 (2004)

DOI: 10.1007/s00454-003-2953-2

\title{
Guest Editor's Foreword
}

This special issue contains a selection of seven papers whose preliminary versions appeared at the Eighteenth Annual Symposium on Computational Geometry held June 5-7, 2002, in Barcelona, Spain.

Chazelle explores the power of subtraction in the computational complexity of range searching. For a "natural" range searching problem, he shows that the arithmetic complexity is $\Theta\left(n^{3 / 2}\right)$ if only addition is allowed, but the complexity drops to $\Theta(n \log n)$ if subtraction is also allowed.

Aichholzer, Aurenhammer and Palop describe the city Voronoi diagram, induced by quickest paths in the $L_{1}$ plane, with streets arranged in the north-south and east-west directions. They explore geometric properties of these diagrams, describe algorithms to construct them and answer quickest-path queries.

Erickson and Har-Peled describe how to obtain a topological disk from a polyhedral manifold by cutting a small subset of the edges. They prove that the optimization problem is NP-complete and describe exact and approximation algorithms.

Cabello, Liu, Mantler and Snoeyink describe algorithms for testing if two given paths are homotopic. For paths consisting of $n$ line segments and obstacles described by $n$ points, their algorithm runs in time $O(n \log n)$ if the paths are simple, and in time $O\left(n^{3 / 2}\right)$ if the paths are self-intersecting.

Koltun and Sharir consider the complexity of the polyhedral Voronoi diagram in three dimensions. They show that the polyhedral Voronoi diagram of $n$ line segments or, more generally, the polyhedral Voronoi diagram of a collection of disjoint polyhedra with a total of $n$ vertices has near quadratic size.

Cheong, Har-Peled, Linial and Matoušek analyze the following one-round Voronoi game in the plane: the first player chooses an $n$-point set $W$ in a unit square; the second player chooses another $n$-point set $B$; each player's payoff is his fraction of the Voronoi diagram of $W \cup B$. They show that the second player can always win at least $\frac{1}{2}+\alpha$, for a constant $\alpha>0$.

Asano, Kirkpatrick and Yap consider approximate solutions to optimization problems. They present an efficient scheme for converting a pseudo $\varepsilon$-approximation algorithm into a true $\varepsilon$-approximation algorithm, and apply this technique to compute $\varepsilon$-approximations for three-dimensional shortest paths and planar rod motion problems.

I wish to thank the authors for contributing their papers to this special issue and the referees for their timely and thorough reviews.

Subhash Suri 